\begin{tabular}{lccr} 
A R C H I V E & OF & M E C H A N I C A L & E N G I N E E R I N G \\
\hline VOL. LXIII & 2016 & Number 2
\end{tabular}

10.1515/meceng-2016-0017

Key words: Shooting method, Harmonic Balance Method, local nonlinearities, periodic solutions, nonsmooth dynamics

\title{
A MIXED SHOOTING - HARMONIC BALANCE METHOD FOR UNILATERALLY CONSTRAINED MECHANICAL SYSTEMS
}

\begin{abstract}
In this paper we present a mixed shooting - harmonic balance method for large linear mechanical systems on which local nonlinearities are imposed. The standard harmonic balance method (HBM), which approximates the periodic solution in frequency domain, is very popular as it is well suited for large systems with many degrees of freedom. However, it suffers from the fact that local nonlinearities cannot be evaluated directly in the frequency domain. The standard HBM performs an inverse Fourier transform, then calculates the nonlinear force in time domain and subsequently the Fourier coefficients of the nonlinear force. The disadvantage of the HBM is that strong nonlinearities are poorly represented by a truncated Fourier series. In contrast, the shooting method operates in time-domain and relies on numerical time-simulation. Set-valued force laws such as dry friction or other strong nonlinearities can be dealt with if an appropriate numerical integrator is available. The shooting method, however, becomes infeasible if the system has many states. The proposed mixed shooting-HBM approach combines the best of both worlds.
\end{abstract}

\section{Introduction}

Finding periodic solutions of mechanical systems is an important task in the design process of machines and mechanical devices. For instance, knowledge of the response of the system to harmonic excitation is essential to obtain information about high cycle fatigue behaviour. In engineering systems local nonlinearities are present due to contact or coupling elements. These local nonlinearities can have a strong impact on the global system behaviour. Therefore, the nonlinearities have to be considered in the design process and must be modelled accurately as well as in a computationally efficient way.

* University of Stuttgart, Institute for Nonlinear Mechanics, Pfaffenwaldring 9, 70569 Stuttgart; E-mails: schreyer@inm.uni-stuttgart.de,leine@inm.uni-stuttgart.de 
The most popular methods to find periodic steady-state responses of nonlinear differential equations are the Harmonic Balance Method (HBM) [1, $2,3,4]$ and the shooting method [5]. The standard HBM approximates the periodic solution in frequency domain and is very popular as it is well suited for large systems with many states. Local nonlinearities cannot be evaluated directly in the frequency domain. The standard HBM performs an inverse Fourier transformation, and then calculates the nonlinear force in time domain and subsequently the Fourier coefficients of the nonlinear force. This procedure is often denoted as the Alternating Frequency Time Method (AFT) [6]. The disadvantage of the HBM is that strong nonlinearities are poorly represented by a truncated Fourier series. Additionally, the restriction of the AFT method to single-valued functions only allows the use of smooth contact laws. In contrast, the shooting method operates in time-domain and relies on numerical time-simulation. Set-valued force laws such as dry friction or other strong nonlinearities can be dealt with if an appropriate numerical integrator is available. The shooting method, however, becomes infeasible if the system has many states. The proposed mixed shooting-HBM approach combines the efficiency of HBM and the accuracy of the shooting method and has therefore many advantages.

In this paper the mixed shooting-HBM (MS-HBM) approach is introduced as a novel method to calculate periodic solutions of forced mechanical systems. Two different variants of the mixed shooting-HBM approach are presented in Section 3.2 and 3.3 respectively. Depending on the position of the local nonlinearities within the mechanical system, the one or the other is better suitable. The more general method of Section 3.2 is tested on a multi-mass oscillator at the end of the paper and is compared to the full HBM and full shooting method. As local nonlinearities, dry friction as well as a hard unilateral constraint are investigated.

\section{Fourier Transformation}

In this paper the Fourier transformation is used and therefore its nomenclature is introduced in this section for an arbitrary time signal $\boldsymbol{x}(t) \in \mathbb{R}^{n}$. The Fourier coefficients

$$
\hat{\boldsymbol{x}}=\mathrm{FFT}(\boldsymbol{x}(t))=\frac{2}{T} \int_{0}^{T} \boldsymbol{J} \boldsymbol{V}(t) \boldsymbol{x}(t) \mathrm{d} t
$$


of the time signal are obtained using the Fast Fourier Transformation (FFT). Hereto,

$$
\boldsymbol{V}(t)=\left(\begin{array}{c}
\boldsymbol{I} \\
\cos (\omega t) \boldsymbol{I} \\
\sin (\omega t) \boldsymbol{I} \\
\vdots \\
\cos \left(n_{H} \omega t\right) \boldsymbol{I} \\
\sin \left(n_{H} \omega t\right) \boldsymbol{I}
\end{array}\right), \quad \boldsymbol{J}=\left(\begin{array}{cccc}
\frac{1}{2} \boldsymbol{I} & \mathbf{0} & \mathbf{0} & \\
\mathbf{0} & \boldsymbol{I} & \mathbf{0} & \ldots \\
\mathbf{0} & \mathbf{0} & \boldsymbol{I} & \\
& \vdots & & \ddots
\end{array}\right)
$$

are defined where $\omega=\frac{2 \pi}{T}$ and $n_{H}$ denote the number of considered harmonics and $\boldsymbol{I}$ is the $n$-dimensional identity matrix. The harmonic oscillation which is described by the Fourier coefficients can be calculated using the matrix multiplications

$$
\boldsymbol{x}_{h}(t)=\operatorname{IFFT}(\hat{\boldsymbol{x}})=\boldsymbol{V}(t)^{\mathrm{T}} \hat{\boldsymbol{x}}
$$

and its time-derivative by

$$
\dot{\boldsymbol{x}}_{h}(t)=\dot{\boldsymbol{V}}(t)^{\mathrm{T}} \hat{\boldsymbol{x}}=\boldsymbol{V}(t)^{\mathrm{T}} \mathbf{\Omega} \hat{\boldsymbol{x}}
$$

where

$$
\boldsymbol{\Omega}=\operatorname{diag}\left(\boldsymbol{\Omega}_{1}, \boldsymbol{\Omega}_{2}, \ldots, \boldsymbol{\Omega}_{n_{H}}\right), \quad \boldsymbol{\Omega}_{k}=\left(\begin{array}{ccc}
\mathbf{0} & \mathbf{0} & \mathbf{0} \\
\mathbf{0} & \mathbf{0} & k \omega \boldsymbol{I} \\
\mathbf{0} & -k \omega \boldsymbol{I} & \mathbf{0}
\end{array}\right)
$$

Equation (3) is often referred to as the inverse Fourier transformation. For the understanding of the following sections note that $\operatorname{IFFT}(\operatorname{FFT}(\boldsymbol{x}(t)))=\boldsymbol{x}(t)$ only holds for a harmonic signal of which all contained harmonics are considered in the ansatz function $\boldsymbol{V}(t)$.

\section{Mixed shooting-HBM approach}

The mixed shooting-HBM approach exploits the local character of the nonlinearities to find periodic solutions of mechanical systems efficiently. The system must therefore be divided into linear and nonlinear subsystems. This can be done with or without an intermediate subsystem leading to the methods of Section 3.2 and 3.3. First the system description is given and subsequently both methods are discussed. 


\subsection{System description}

We consider a Lagrangian system of the form

$$
\boldsymbol{M} \ddot{\boldsymbol{q}}(t)+\boldsymbol{C} \dot{\boldsymbol{q}}(t)+\boldsymbol{K} \boldsymbol{q}(t)=\boldsymbol{f}_{\mathrm{ex}}(t)+\boldsymbol{f}_{\mathrm{nl}}(\boldsymbol{q}(t), \dot{\boldsymbol{q}}(t)),
$$

where $f_{\mathrm{nl}}$ contains the nonlinear forces and $f_{\mathrm{ex}}(t)=f_{\mathrm{ex}}(t+T)$ is the periodic forcing. We assume that the system consists of a linear and a nonlinear subsystem with the generalized coordinates

$$
\boldsymbol{q}=\left(\begin{array}{l}
\boldsymbol{q}_{N} \\
\boldsymbol{q}_{L}
\end{array}\right)
$$

that the nonlinear forces only act on the nonlinear subsystem, and that the system matrices $\boldsymbol{M}, \boldsymbol{C}$ and $\boldsymbol{K}$ have the following structure

$$
\boldsymbol{M}=\left(\begin{array}{ll}
\boldsymbol{M}_{N N} & \boldsymbol{M}_{N L} \\
\boldsymbol{M}_{L N} & \boldsymbol{M}_{N N}
\end{array}\right), \quad \boldsymbol{f}_{\mathrm{nl}}(\boldsymbol{q}, \dot{\boldsymbol{q}})=\left(\begin{array}{c}
\boldsymbol{f}_{\mathrm{nl}, N}\left(\boldsymbol{q}_{N}, \dot{\boldsymbol{q}}_{N}\right) \\
\mathbf{0}
\end{array}\right) .
$$

The nonlinear subsystem is subjected to nonlinear forces, which only depend on its own positions and velocities e.g. the three DOF oscillator shown in Fig. 1.

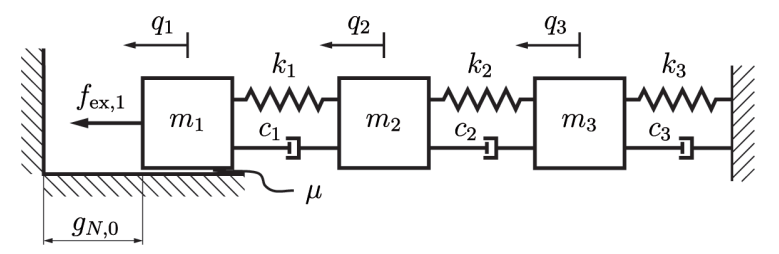

Fig. 1. Three DOF oscillator with dry friction and unilateral constraint

\subsection{MS-HBM with a linear and a nonlinear subsystem}

The reduction into two subsystems can reduce the computational effort for systems for which the relationship $\operatorname{dim}\left(\boldsymbol{q}_{L}\right) \gg \operatorname{dim}\left(\boldsymbol{q}_{N}\right)$ between the dimensions of the subsystems holds. The linear subsystem which is described by

$$
\boldsymbol{M}_{L L} \ddot{\boldsymbol{q}}_{L}+\boldsymbol{C}_{L L} \dot{\boldsymbol{q}}_{L}+\boldsymbol{K}_{L L} \boldsymbol{q}_{L}+\boldsymbol{f}_{c}=\boldsymbol{f}_{\mathrm{ex}, L}
$$

with the dynamic coupling force between both subsystem

$$
\boldsymbol{f}_{c}=\boldsymbol{M}_{L N} \ddot{\boldsymbol{q}}_{N}+\boldsymbol{C}_{L N} \dot{\boldsymbol{q}}_{N}+\boldsymbol{K}_{L N} \boldsymbol{q}_{N}
$$


is approximated using a Ritz-Galerkin Method. The ansatz for the displacement field is chosen as

$$
\mathcal{U}_{n_{H}}=\left\{\boldsymbol{q}_{L}(t) \in \mathcal{U} \mid \boldsymbol{q}_{L}(t)=\boldsymbol{V}_{L}(t)^{\mathrm{T}} \hat{\boldsymbol{q}}_{L}\right\},
$$

where $\mathcal{U}$ is the space of absolutely continuous functions. In other words, due to the Galerkin approach, the motion of the linear subsystem is approximated by a truncated Fourier series

$$
\boldsymbol{q}_{L}(t)=\hat{\boldsymbol{q}}_{L}^{0}+\sum_{k=1}^{n_{H}} \hat{\boldsymbol{q}}_{L}^{c, k} \cos (k \omega t)+\hat{\boldsymbol{q}}_{L}^{s, k} \sin (k \omega t)=\boldsymbol{V}_{L}(t)^{\mathrm{T}} \hat{\boldsymbol{q}}_{L} .
$$

The equality (12) can be envisaged as a constraint on the system and will be referred to as the harmonic constraint on the linear subsystem.

According to the Galerkin method the corresponding virtual displacement field

$$
\mathcal{V}_{n_{H}}=\left\{\delta \boldsymbol{q}_{L}(t) \mid \delta \boldsymbol{q}_{L}(t)=\boldsymbol{V}_{L}(t)^{\mathrm{T}} \delta \hat{\boldsymbol{q}}_{L}\right\}
$$

is described using the same ansatz functions. To obtain the harmonically constrained subsystem one requires that the virtual work of the linear subsystem

$$
\delta \boldsymbol{W}=\int_{0}^{T} \delta \boldsymbol{q}_{L}^{\mathrm{T}}\left(\boldsymbol{M}_{L L} \ddot{\boldsymbol{q}}_{L}+\boldsymbol{C}_{L L} \dot{\boldsymbol{q}}_{L}+\boldsymbol{K}_{L L} \boldsymbol{q}_{L}+\boldsymbol{f}_{c}-\boldsymbol{f}_{\mathrm{ex}, L}\right) \mathrm{d} t=0,
$$

vanishes for all virtual displacements $\delta \boldsymbol{q}_{L} \in \mathcal{V}_{n_{H}}$ and $\boldsymbol{q}_{L} \in \mathcal{U}_{n_{H}}$. From (14) with (11) and (13) the virtual work can be described as

$$
\begin{aligned}
\delta \boldsymbol{W}= & \delta \hat{\boldsymbol{q}}_{L}^{\mathrm{T}} \int_{0}^{T}\left(\boldsymbol{V}_{L} \boldsymbol{M}_{L L} \boldsymbol{\Omega}^{2} \boldsymbol{V}_{L}^{\mathrm{T}}+\boldsymbol{V}_{L} \boldsymbol{C}_{L L} \boldsymbol{\Omega} \boldsymbol{V}_{L}^{\mathrm{T}}+\boldsymbol{V}_{L} \boldsymbol{K}_{L L} \boldsymbol{V}_{L}^{\mathrm{T}}\right) \mathrm{d} t \hat{\boldsymbol{q}}_{L}+ \\
& +\delta \hat{\boldsymbol{q}}_{L}^{\mathrm{T}} \int_{0}^{T}\left(\boldsymbol{V}_{L} \boldsymbol{f}_{c}\right) \mathrm{d} t-\delta \hat{\boldsymbol{q}}_{L}^{\mathrm{T}} \int_{0}^{T}\left(\boldsymbol{V}_{L} \boldsymbol{f}_{\mathrm{ex}, L}\right) \mathrm{d} t=0 \quad \forall \delta \hat{\boldsymbol{q}}_{L} .
\end{aligned}
$$

Using (1) and by pre-multiplying (15) with $\frac{2}{T} J$, the last two force terms in (15) can be interpreted as the Fourier coefficients $\hat{f}_{c}$ and $\hat{f}_{\mathrm{ex}, L}$ of their respective time signals. The dynamics of the linear subsystem can therefore be described in frequency domain as

$$
\boldsymbol{H}_{L L} \hat{\boldsymbol{q}}_{L}+\hat{\boldsymbol{f}}_{c}-\hat{\boldsymbol{f}}_{\mathrm{ex}, L}=\mathbf{0},
$$

where $\hat{\boldsymbol{q}}_{L}$ represent the Fourier coefficients of the linear subsystem and

$$
\boldsymbol{H}_{L L}=\frac{2}{T} \int_{0}^{T} \boldsymbol{J} \boldsymbol{V}_{L} \boldsymbol{M}_{L L} \boldsymbol{\Omega}^{2} \boldsymbol{V}_{L}^{\mathrm{T}}+\boldsymbol{J} \boldsymbol{V}_{L} \boldsymbol{C}_{L L} \boldsymbol{\Omega} \boldsymbol{V}_{L}^{\mathrm{T}}+\boldsymbol{J} \boldsymbol{V}_{L} \boldsymbol{K}_{L L} \boldsymbol{V}_{L}^{\mathrm{T}} \mathrm{d} t
$$


is the dynamic stiffness matrix. The Fourier coefficients of the coupling force resulting from the nonlinear system are given by

$$
\begin{aligned}
\hat{\boldsymbol{f}_{c}} & =\frac{2}{T} \int_{0}^{T} \boldsymbol{J} \boldsymbol{V}_{L} \boldsymbol{f}_{c}(t) \mathrm{d} t= \\
& =\frac{2}{T} \int_{0}^{T} \boldsymbol{J} \boldsymbol{V}_{L} \boldsymbol{f}_{c, h}(t) \mathrm{d} t
\end{aligned}
$$

where $f_{c, h}(t)$ is the harmonic part of the coupling force $f_{c}(t)$. The harmonic part $f_{c, h}(t)$ only depends on the harmonic part of the motion of the nonlinear subsystem which can be expressed in the Fourier coefficients $\hat{\boldsymbol{q}}_{N}$, i.e.

$$
\begin{aligned}
\boldsymbol{f}_{c, h}(t) & =\boldsymbol{M}_{L N} \ddot{\boldsymbol{q}}_{N, h}+\boldsymbol{C}_{L N} \dot{\boldsymbol{q}}_{N, h}+\boldsymbol{K}_{L N} \boldsymbol{q}_{N, h}= \\
& =\boldsymbol{M}_{L N} \boldsymbol{\Omega}^{2} \boldsymbol{V}_{N}^{\mathrm{T}} \hat{\boldsymbol{q}}_{N}+\boldsymbol{C}_{L N} \boldsymbol{\Omega} \boldsymbol{V}_{N}^{\mathrm{T}} \hat{\boldsymbol{q}}_{N}+\boldsymbol{K}_{L N} \boldsymbol{V}_{N}^{\mathrm{T}} \hat{\boldsymbol{q}}_{N} .
\end{aligned}
$$

Hence, the Fourier coefficients of the coupling force can be expressed as

$$
\begin{aligned}
\hat{\boldsymbol{f}}_{c} & =\frac{2}{T} \int_{0}^{T} \boldsymbol{J} \boldsymbol{V}_{L} \boldsymbol{M}_{L N} \boldsymbol{\Omega}^{2} \boldsymbol{V}_{N}^{\mathrm{T}}+\boldsymbol{J} \boldsymbol{V}_{L} \boldsymbol{C}_{L N} \boldsymbol{\Omega} \boldsymbol{V}_{N}^{\mathrm{T}}+\boldsymbol{J} \boldsymbol{V}_{L} \boldsymbol{K}_{L N} \boldsymbol{V}_{N}^{\mathrm{T}} \mathrm{d} t \hat{\boldsymbol{q}}_{N}= \\
& =\boldsymbol{H}_{L N} \hat{\boldsymbol{q}}_{N}
\end{aligned}
$$

The harmonic constraint can be viewed as a Galerkin projection of the dynamics of the linear subsystem on the chosen harmonic basis (which is of course an approximation of the dynamics). The nonlinear subsystem, which is not constrained to oscillate harmonically, acts as external forcing $f_{c}(t)$ on the linear subsystem. The inharmonic part of this forcing, e.g. $f_{c}(t)-f_{c, h}(t)$, is counterbalanced by the perfect constraint force which enforces the harmonic constraint (12). Similarly, the inharmonic part of the excitation force $f_{\text {ex }, L}(t)$ is absorbed by the harmonic constraint and its contribution to (16) is given by

$$
\hat{f}_{\mathrm{ex}, L}=\frac{2}{T} \int_{0}^{T} \boldsymbol{J} \boldsymbol{V}_{L} \boldsymbol{f}_{\mathrm{ex}, L} \mathrm{~d} t .
$$

Using (16), we obtain the linear relation

$$
\hat{\boldsymbol{q}}_{L}=\boldsymbol{H}_{L L}^{-1}\left(\hat{\boldsymbol{f}}_{\mathrm{ex}, L}-\boldsymbol{H}_{L N} \hat{\boldsymbol{q}}_{N}\right)
$$

between the Fourier coefficients of the linear subsystem $\hat{\boldsymbol{q}}_{L}$ and the Fourier coefficients of the nonlinear subsystem $\hat{\boldsymbol{q}}_{N}$. The dynamic stiffness matrices $\boldsymbol{H}_{i j}$ result from Equation (17) and (20)

$$
\boldsymbol{H}_{i j}=\operatorname{diag}\left(\boldsymbol{H}_{i j, 0}, \boldsymbol{H}_{i j, 1}, \ldots \boldsymbol{H}_{i j, n_{H}}\right)
$$

where the diagonal entries 


$$
\boldsymbol{H}_{i j, k}=\left(\begin{array}{cc}
-\boldsymbol{M}_{i j}(k \omega)^{2}+\boldsymbol{K}_{i j} & \boldsymbol{C}_{i j} k \omega \\
-\boldsymbol{C}_{i j} k \omega & -\boldsymbol{M}_{i j}(k \omega)^{2}+\boldsymbol{K}_{i j}
\end{array}\right)
$$

are given for each considered harmonic oscillation. For given $\hat{\boldsymbol{q}}_{N}$ the equation of motion of the linear subsystem is therefore completely described by (22). The equation of motion of the nonlinear subsystem

$$
\begin{array}{r}
\boldsymbol{M}_{N N} \ddot{\boldsymbol{q}}_{N}+\boldsymbol{C}_{N N} \dot{\boldsymbol{q}}_{N}+\boldsymbol{K}_{N N} \boldsymbol{q}_{N}= \\
=-\boldsymbol{M}_{N L} \ddot{\boldsymbol{q}}_{L}-\boldsymbol{C}_{N L} \dot{\boldsymbol{q}}_{L}-\boldsymbol{K}_{N L} \boldsymbol{q}_{L}-\boldsymbol{f}_{\mathrm{ex}, N}+\boldsymbol{f}_{\mathrm{nl}, N}
\end{array}
$$

is described in time domain. If $\hat{\boldsymbol{q}}_{N}$ is known, then the time-evolution $\boldsymbol{q}_{L}(t)$ and its derivatives are given using (22) together with (12). The influence of the linear subsystem can therefore be considered as external forcing. To ensure that both subsystems oscillate correspondingly a connectivity condition must be considered in the residuum function. Hence, only the nonlinear subsystem must be described as differential equation, which has to be solved for $\boldsymbol{q}_{N}(t)$ using numerical time integration. In particular, if the nonlinear force $f_{\mathrm{nl}, N}$ is a dry friction force or, more generally, described by a set-valued force law, then dedicated time-integration schemes such as timestepping methods [7,8] have to be used. Here it should be noted, that the system (6) and consequently (25) turns into a differential inclusion if a set-valued force law is considered.

A periodic solution of the complete system can be represented by the trajectory $\boldsymbol{q}_{N}(t)$ on the interval $0 \leq t \leq T$ and by the Fourier coefficients $\hat{\boldsymbol{q}}_{N}$, as $\hat{\boldsymbol{q}}_{L}$ is expressed by (22). The initial condition $\boldsymbol{q}_{N}(0)$ and $\dot{\boldsymbol{q}}_{N}(0)$ together with $\boldsymbol{q}_{L}(t)=\boldsymbol{V}_{L}(t)^{\mathrm{T}} \hat{\boldsymbol{q}}_{L}$ allow to construct $\boldsymbol{q}_{N}(t)$ over one period. The vector of unknowns

$$
\boldsymbol{x}=\left(\begin{array}{c}
\hat{\boldsymbol{q}}_{N} \\
\boldsymbol{q}_{N}(0) \\
\dot{\boldsymbol{q}}_{N}(0)
\end{array}\right)
$$

therefore fully represents a periodic solution of the system. Similar to a shooting method, we require for the nonlinear subsystem the periodicity conditions $\boldsymbol{q}_{N}(T)-\boldsymbol{q}_{N}(0)=\mathbf{0}$ and $\dot{\boldsymbol{q}}_{N}(T)-\dot{\boldsymbol{q}}_{N}(0)=\mathbf{0}$, where the state at $t=T$ is obtained through numerical time-integration of (25). The periodicity condition of the linear subsystem is given in frequency domain by (22). Hence, we seek a periodic solution by finding a zero of the nonlinear function

$$
\boldsymbol{f}_{R}(\boldsymbol{x})=\left(\begin{array}{c}
\hat{\boldsymbol{q}}_{N}-\mathrm{FFT}\left(\boldsymbol{q}_{N}(t)\right) \\
\boldsymbol{q}_{N}(T)-\boldsymbol{q}_{N}(0) \\
\dot{\boldsymbol{q}}_{N}(T)-\dot{\boldsymbol{q}}_{N}(0)
\end{array}\right) .
$$


Note that $\operatorname{FFT}\left(\boldsymbol{q}_{N}(t)\right)$ is the Fourier transformation

$$
\operatorname{FFT}\left(\boldsymbol{q}_{N}(t)\right)=\frac{2}{T} \int_{0}^{T} \boldsymbol{J} \boldsymbol{V}_{N} \boldsymbol{q}_{N}(t) \mathrm{d} t
$$

of the solution of the differential equation (25) and $\hat{\boldsymbol{q}}_{N}$ are the Fourier coefficients which represent the dynamical behaviour of the linear subsystem in the vector of unknowns $\boldsymbol{x}$ through (22). If the connectivity condition $\hat{\boldsymbol{q}}_{N}-\operatorname{FFT}\left(\boldsymbol{q}_{N}(t)\right)=\mathbf{0}$ holds, then the linear subsystem is oscillating in correspondence to the movement of the nonlinear subsystem. Obviously, due to the harmonic approximation, the connectivity condition between both subsystems leads to an approximated solution of the linear and the nonlinear subsystem. The periodic solution converges to the exact solution for an increasing number of considered harmonic oscillations. The zeros of $\boldsymbol{f}_{R}(\boldsymbol{x})$ can be solved with a Newton-type method by iterating

$$
\boldsymbol{x}^{i+1}=\boldsymbol{x}^{i}-\left(\frac{\partial \boldsymbol{f}_{R}}{\partial \boldsymbol{x}}\right)^{-1} \boldsymbol{f}_{R}\left(\boldsymbol{x}^{i}\right)
$$

where the Jacobian is obtained through finite differences. The calculation procedure of this method is depicted in Fig. 2.

\subsection{MS-HBM using an intermediate subsystem}

For the second approach System (6) must be divided into three subsystems. The first subsystem is subjected to the nonlinear force and is coupled through the linear Subsystem 2 to the third subsystem which has to be linear as well. For the second approach the system matrices must therefore exist in the form

$$
\boldsymbol{M}=\left(\begin{array}{ccc}
\boldsymbol{M}_{11} & \boldsymbol{M}_{12} & \mathbf{0} \\
\boldsymbol{M}_{21} & \boldsymbol{M}_{22} & \boldsymbol{M}_{23} \\
\mathbf{0} & \boldsymbol{M}_{32} & \boldsymbol{M}_{33}
\end{array}\right), \boldsymbol{f}_{\mathrm{nl}}(\boldsymbol{q}, \dot{\boldsymbol{q}})=\left(\begin{array}{c}
\boldsymbol{f}_{\mathrm{nl}, N}\left(\boldsymbol{q}_{1}, \dot{\boldsymbol{q}}_{1}\right) \\
\mathbf{0} \\
\mathbf{0}
\end{array}\right), \boldsymbol{q}=\left(\begin{array}{l}
\boldsymbol{q}_{1} \\
\boldsymbol{q}_{2} \\
\boldsymbol{q}_{3}
\end{array}\right) .
$$

This method is suitable if the relation between the dimensions of the subsystems

$$
\operatorname{dim}\left(\boldsymbol{q}_{3}\right) \gg \operatorname{dim}\left(\boldsymbol{q}_{1}\right)>\operatorname{dim}\left(\boldsymbol{q}_{2}\right)
$$

holds. Using the first approach Subsystem 1 and 3 do not have to be uncoupled since the system is not restricted to condition (31). The first approach is therefore more general than the method described in this section. If the relation (31) between the dimensions is fulfilled, then the approach using an intermediate subsystem can be beneficial. This may for instance be the case 


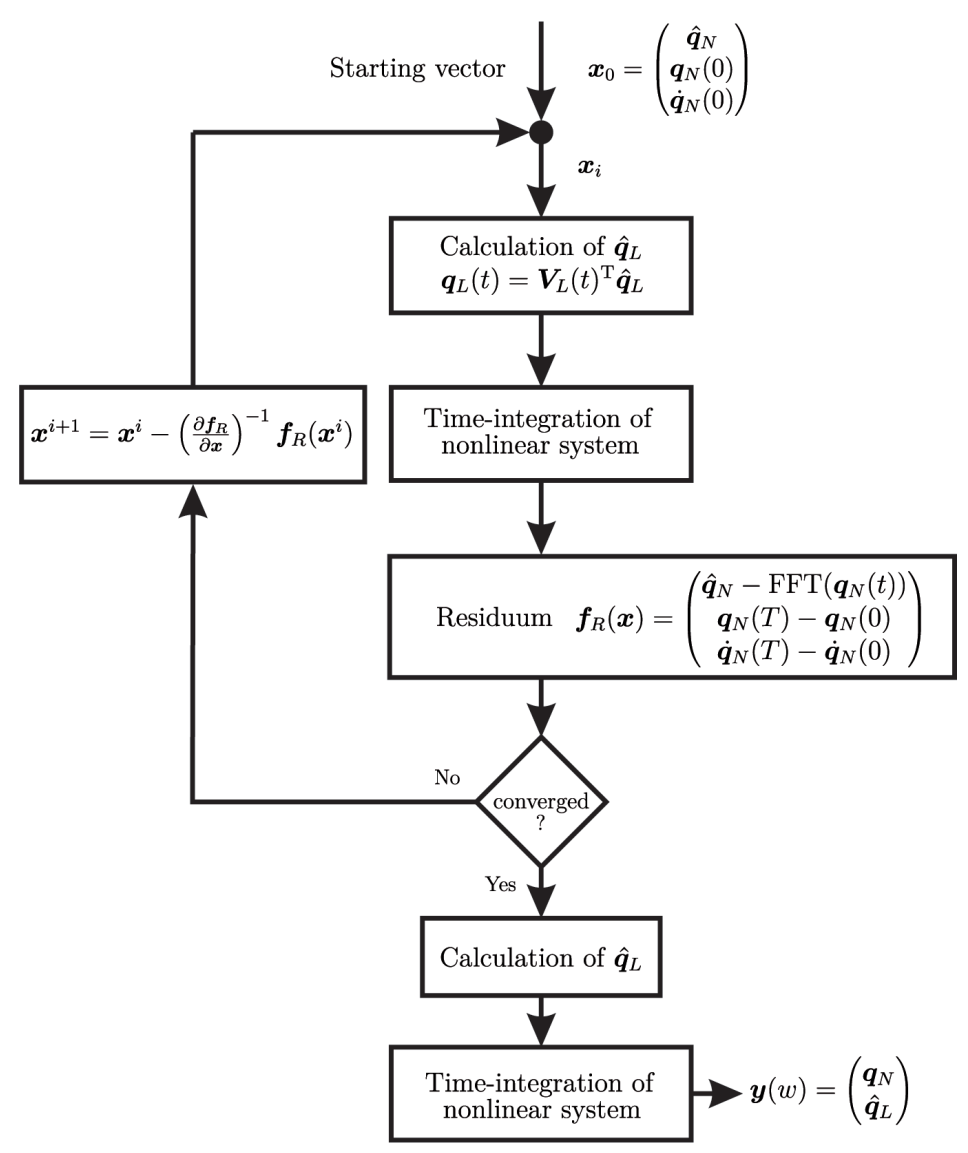

Fig. 2. Calculation scheme of MS-HBM with linear and nonlinear subsystem

for an extension of the system depicted in Fig. 1 to a chain of $n$ masses, where the first $n_{1}$ masses are subjected to friction and the mass $n_{1}+1$ acts as intermediate subsystem.

For Subsystem 2 and 3 we use the Galerkin approach, as before for the linear subsystem, and impose perfect constraints on the system which force the response to be harmonic of the form

$\boldsymbol{q}_{2}(t)=\hat{\boldsymbol{q}}_{2}^{0}+\sum_{k=1}^{n_{H}} \hat{\boldsymbol{q}}_{2}^{c, k} \cos k \omega t+\hat{\boldsymbol{q}}_{2}^{s, k} \sin k \omega t=\boldsymbol{V}_{2}(t)^{\mathrm{T}} \hat{\boldsymbol{q}}_{2}, \quad \boldsymbol{q}_{3}(t)=\boldsymbol{V}_{3}(t)^{\mathrm{T}} \hat{\boldsymbol{q}}_{3}$.

The motion $\boldsymbol{q}_{1}(t)$ of Subsystem 1 is described in time domain and is not constrained to be harmonic. The Fourier coefficients of the generalized coordinates $\boldsymbol{q}_{1}(t)$ are obtained from

$$
\hat{\boldsymbol{q}}_{1}=\frac{2}{T} \int_{0}^{T} \boldsymbol{J} \boldsymbol{V}_{1}(t) \boldsymbol{q}_{1}(t) \mathrm{d} t
$$


The equations of motion of Subsystem 2 and 3 can therefore be expressed in frequency domain as

$$
\begin{aligned}
\boldsymbol{H}_{21} \hat{\boldsymbol{q}}_{1}+\boldsymbol{H}_{22} \hat{\boldsymbol{q}}_{2}+\boldsymbol{H}_{23} \hat{\boldsymbol{q}}_{3} & =\hat{\boldsymbol{f}}_{\mathrm{ex} 2}, \\
\boldsymbol{H}_{32} \hat{\boldsymbol{q}}_{2}+\boldsymbol{H}_{33} \hat{\boldsymbol{q}}_{3} & =\hat{\boldsymbol{f}}_{\mathrm{ex} 3},
\end{aligned}
$$

where $\boldsymbol{H}_{i j}$ are the dynamic stiffness matrices. Using (34) the Fourier coefficients $\hat{\boldsymbol{q}}_{3}$ can be expressed in $\hat{\boldsymbol{q}}_{2}$ as

$$
\hat{\boldsymbol{q}}_{3}=\boldsymbol{H}_{33}^{-1}\left(\hat{\mathrm{f}}_{\mathrm{ex} 3}-\boldsymbol{H}_{32} \hat{\boldsymbol{q}}_{2}\right)
$$

and can therefore be eliminated from the equations of motion in frequency domain, i.e.

$$
\boldsymbol{H}_{21} \hat{\boldsymbol{q}}_{1}+\left(\boldsymbol{H}_{22}-\boldsymbol{H}_{23} \boldsymbol{H}_{33}^{-1} \boldsymbol{H}_{32}\right) \hat{\boldsymbol{q}}_{2}=\hat{\boldsymbol{f}}_{\mathrm{ex} 2}-\boldsymbol{H}_{23} \boldsymbol{H}_{33}^{-1} \hat{\boldsymbol{f}}_{\mathrm{ex} 3} .
$$

The equations of motion of Subsystem 1 are nonlinear and are simulated in time-domain. For known $\hat{\boldsymbol{q}}_{2}$ one can calculate its time-domain representation $\boldsymbol{q}_{2}(t)$ and its derivatives and solve the differential equation for $\boldsymbol{q}_{1}(t)$

$$
\begin{aligned}
& \boldsymbol{M}_{11} \ddot{\boldsymbol{q}}_{1}(t)+\boldsymbol{C}_{11} \dot{\boldsymbol{q}}_{1}(t)+\boldsymbol{K}_{11} \boldsymbol{q}_{1}(t)=-\left(\boldsymbol{M}_{12} \ddot{\boldsymbol{q}}_{2}(t)+\right. \\
& \left.+\boldsymbol{C}_{12} \dot{\boldsymbol{q}}_{2}(t)+\boldsymbol{K}_{12} \boldsymbol{q}_{2}(t)\right)+\boldsymbol{f}_{\mathrm{ex} 1}(t)+\boldsymbol{f}_{\mathrm{n} 11}\left(\boldsymbol{q}_{1}(t), \dot{\boldsymbol{q}}_{1}(t)\right)
\end{aligned}
$$

using numerical integration techniques. A periodic solution is completely described by the vector of unknowns

$$
\boldsymbol{x}=\left(\begin{array}{c}
\hat{\boldsymbol{q}}_{2} \\
\boldsymbol{q}_{1}(0) \\
\dot{\boldsymbol{q}}_{1}(0)
\end{array}\right)
$$

We require for Subsystem 1 the periodicity conditions $\boldsymbol{q}_{1}(T)-\boldsymbol{q}_{1}(0)=\mathbf{0}$ and $\dot{\boldsymbol{q}}_{1}(T)-\dot{\boldsymbol{q}}_{1}(0)=\mathbf{0}$, where the state at $t=T$ is obtained through numerical time-integration of (37). The periodicity conditions of Subsystems 2 and 3 are given in frequency domain by (36) and (35). The residuum function to find a periodic solution

$$
\boldsymbol{f}_{R}(\boldsymbol{x})=\left(\begin{array}{c}
\boldsymbol{H}_{21} \hat{\boldsymbol{q}}_{1}+\left(\boldsymbol{H}_{22}-\boldsymbol{H}_{23} \boldsymbol{H}_{33}^{-1} \boldsymbol{H}_{32}\right) \hat{\boldsymbol{q}}_{2}-\hat{\boldsymbol{f}}_{\mathrm{ex} 2}+\boldsymbol{H}_{23} \boldsymbol{H}_{33}^{-1} \hat{\boldsymbol{f}}_{\mathrm{ex} 3} \\
\boldsymbol{q}_{1}(T)-\boldsymbol{q}_{1}(0) \\
\dot{\boldsymbol{q}}_{1}(T)-\dot{\boldsymbol{q}}_{1}(0)
\end{array}\right)
$$

is defined by (36) and the periodicity condition of Subsystem 1. The zeros of $\boldsymbol{f}_{R}(\boldsymbol{x})$ can be solved with a Newton-type method by iterating

$$
\boldsymbol{x}^{i+1}=\boldsymbol{x}^{i}-\left(\frac{\partial \boldsymbol{f}_{R}}{\partial \boldsymbol{x}}\right)^{-1} \boldsymbol{f}_{R}\left(\boldsymbol{x}^{i}\right)
$$




\section{Numerical examples}

The three DOF-oscillator (Fig. 1) is used as a numerical benchmark to compare the MS-HBM approach (method of Section 3.2) with the full shooting method and the full HBM, in both computation effort as well as accuracy. The system is inertially decoupled due to its diagonal mass matrix. It is important to note, that the MS-HBM is not restricted to inertially decoupled systems. However, the presence of inertial coupling may necessitate to use a large number $n_{H}$ of harmonics to arrive at a sufficiently accurate approximation of the periodic solution. Another way to improve the accuracy could be to expand the nonlinear subsystem in order to describe a larger part of the system in time domain. Since the full shooting method and the MS-HBM approach solve the nonlinear subsystem as a nonlinear differential inclusion, modern time-stepping methods with a set-valued force law are used for both methods. In contrast to the full shooting method and MS-HBM, the standard HBM with alternating frequency time approach only calculates the nonlinear force in time domain which makes it impossible to use the same contact model. Two types of contacts are considered separately in this work to compare the different methods for a system which is subjected to friction or to a completely elastic unilateral constraint.

\subsection{System with friction}

First, the different methods are investigated for a system under influence of dry friction. Using the MS-HBM or the shooting approach a set-valued force law can be used within the concept of (measure) differential inclusions. The friction force is expressed by the set-valued relationship

$$
-\lambda_{T} \in \mu F_{N} \operatorname{Sign}\left(\gamma_{T}\right)=\left\{\begin{array}{cc}
\mu F_{N}, & \gamma_{T}>0 \\
{[-1,1] \mu F_{N},} & \gamma_{T}=0 \\
-\mu F_{N}, & \gamma_{T}<0
\end{array}\right.
$$

The parameters $\mu$ and $F_{N}$ are the friction coefficient and normal load, respectively. This friction model cannot be used for the HBM. To compare the methods in a most suitable way, the friction force for the HBM is approximated using an arctangent function

$$
-\lambda_{T}^{\text {smooth }}=\mu F_{N} \frac{2}{\pi} \arctan \left(\kappa \gamma_{T}\right)
$$

being a smoothed approximation of (41). The approximation (42) tends to the set-valued force law (41) for large values of the smoothing parameter $\kappa$. 
In Fig. 3 the displacements of the system calculated with all three methods for the period $T=10 \mathrm{~s}$ are shown. During this period the first mass shows a pronounced stick-slip behaviour. Though for the HBM 20 harmonics and for the MS-HBM only 3 harmonics are considered, the mixed method approximates much better the results of the full shooting method. The smoothing parameter is chosen preferably high $(\kappa=800)$. The mixed and full shooting method employ the set-valued description (41) of the friction law and can therefore describe stiction precisely. The HBM, however, not only uses the smoothed friction law (42) but also uses harmonic shape functions to approximate the friction force which leads to a poor description of this force. In contrast, the MS-HBM describes the nonlinear subsystem in time domain and approximates only the coupling between both subsystems with harmonic shape functions.
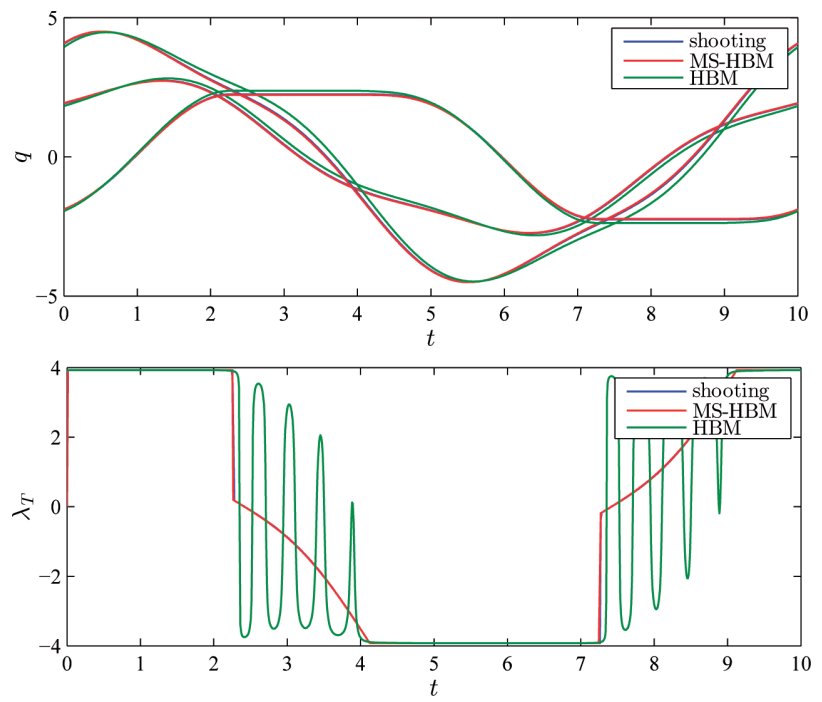

Fig. 3. Displacement and friction force for a periodic solution with period time $T=10 \mathrm{~s}$ of the three DOF oscillator with dry friction

The MS-HBM approach becomes more advantageous than the full shooting method if the dimension of the linear subsystem is much larger than that of the nonlinear subsystem. To demonstrate this, the linear subsystem is extended with additional masses. This expanded model is used to compare the full HBM, the full shooting and the mixed approach. The excitation force is chosen as $f_{\mathrm{ex} i}=0$ for $i=1 \ldots n-1$ and $f_{\mathrm{ex} n}=5 \cos (\omega t)$. The methods are compared for one excitation frequency in computation effort and accuracy. To start the calculation for a specific excitation frequency, a starting guess for the first iteration is needed. However, the methods iterate in different unknowns and the same starting guess can therefore not be given. The HBM 
needs an initial guess for the Fourier coefficients of the nonlinear subsystem, whereas the starting guess for the MS-HBM consists of the state of the nonlinear subsystem and Fourier coefficients which describe the motion of the linear subsystem through (22). However, to provide comparable starting guesses, solutions for an excitation frequency close to the actual frequency are used as starting vectors for the iterative loops of the respective approximation methods. This choice seems reasonable, since this is done as well if a frequency response diagram is calculated.

In Fig. 4 the relative error of the amplitude of the first and $n$th mass and the calculation effort is shown for different numbers of considered harmonics $n_{H}$. Both ratios are with respect to the full shooting method, which is chosen as reference as it is almost exact.

The results show that the computation effort for a moderate accuracy can be reduced drastically by using the MS-HBM approach. Compared to the HBM, the mixed approach shows for all values of $n_{H}$ more accurate results. The horizontal plateau of the relative error of the mixed method can be explained by the limited resolution of the used Fourier transformation and the integration schemes. Therefore, an increase in the number of harmonics can only decrease the error down to a problem dependent minimum value.

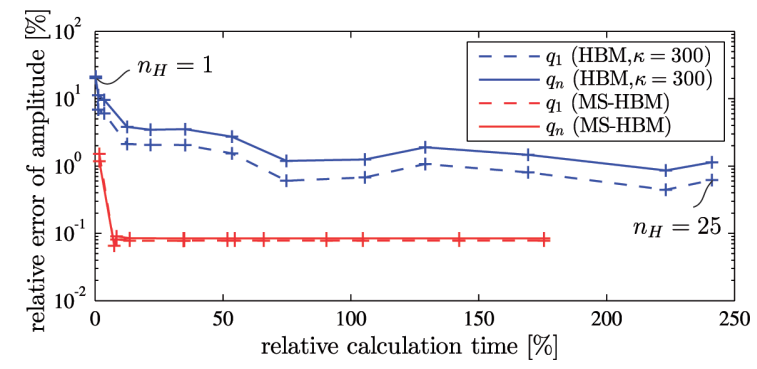

Fig. 4. Work-precision-diagram of the HBM and the mixed shooting-HBM approach in relation to full shooting for a system of $n=30$ masses with friction and different numbers of considered harmonics $\left(n_{H}=1,3, \ldots, 25\right)$

The used parameters for the calculations of the system with friction are summarized in Table 1.

Selected parameters for the system with friction

\begin{tabular}{|c|c|c|c|c|c|c|}
\hline parameter & $m_{i}$ & $k_{i}$ & $c_{i}$ & $\mu$ & $\omega$ & $f_{\mathrm{ex}, 30}$ \\
\hline value & 1 & 1 & 0 & 0.8 & $\frac{1}{5} \pi$ & $5 \cos (\omega t)$ \\
\hline
\end{tabular}




\subsection{System with unilateral constraint}

In the second example, the friction force on the first mass is replaced by a unilateral constraint. The unilateral constraint is modelled within the concept of measure differential inclusions using the hard contact law

$$
0 \leq g_{N} \perp \lambda_{N} \geq 0
$$

where $g_{N}$ is the contact distance $\left(g_{N}=g_{N, 0}-q_{1}\right)$ and $\lambda_{N}$ represents the contact force. The Newtonian impact law is expressed through the inequality complementarity

$$
0 \leq \gamma_{N}^{+}+e_{N} \gamma_{N}^{-} \perp \Lambda_{N} \geq 0 \quad \text { with } 0 \leq e_{N} \leq 1,
$$

with the post-and pre-impact relative velocities $\gamma_{N}^{+}$and $\gamma_{N}^{-}$, the contact impulse $\Lambda_{N}$ and the restitution coefficient $e_{N}$. For a more detailed description of the contact law and impact law see e.g. [8]. The concept of measure differential inclusions with set-valued contact and impact law can only be used for the MS-HBM and full shooting method. As discussed at the beginning of Section 4, the HBM only allows a single-valued contact law. Therefore, the contact for the HBM is modelled using a one-sided springdamper element

$$
-\lambda_{N}^{\text {penalty }}=\left\{\begin{array}{cc}
k_{c} g_{N}+d_{c} \gamma_{N} & g_{N} \leq 0 \\
0 & g_{N}>0
\end{array}\right.
$$

which is in fact a nonsmooth contact law for $d_{c}>0$ as the contact force jumps at collision time instants. The equivalent restitution coefficient $e_{N}$ for a specific one-sided spring-damper element can be calculated following Brogliato [9]. Since only a non-dissipative elastic contact $\left(e_{N}=1\right)$ is used in this work, $d_{c}$ is taken as zero and the model tends to the hard contact if $k_{c}$ is tending to infinity. In Fig. 5, the displacements of a five DOF oscillator with a contact distance $\left(g_{N, 0}=0.1\right)$ at the first mass for the HBM, MS-HBM and the full shooting method are depicted. The used parameters are summarized in Table 2. Figure 6 shows the velocity of the first mass. Like before for the system with dry friction, the HBM has difficulties to approximate the jump in the velocity of the first mass at the collision time-instant $(t=0.55)$, although the contact stiffness $k_{c}$ is chosen relatively high. The mixed and full shooting method show a true velocity jump whereas the HBM only gives a rough approximation of this phenomenon.

To demonstrate the efficiency of the MS-HBM for the system with unilateral constraint, it is extended to the size of 50 masses. The investigated 


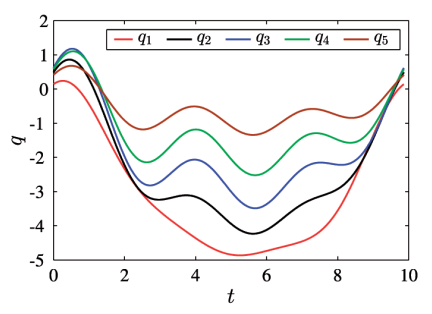

a) $\operatorname{HBM} n_{H}=3$

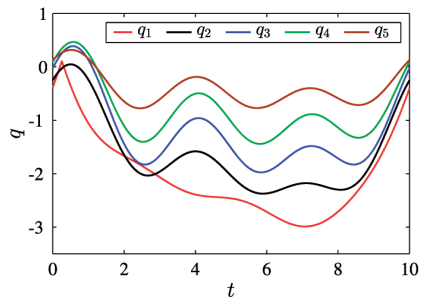

c) MS-HBM $n_{H}=3$

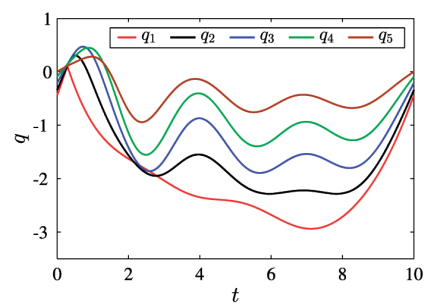

e) full shooting

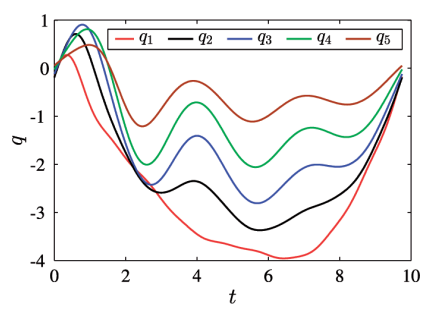

b) $\operatorname{HBM} n_{H}=9$

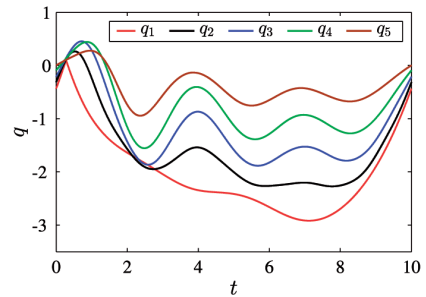

d) MS-HBM $n_{H}=9$

Fig. 5. Displacements of a five DOF oscillator with impact calculated with the different methods

Selected parameters for the system with impact

\begin{tabular}{|c|c|c|c|c|c|c|c|c|}
\hline parameter & $m_{1}$ & $m_{2-5}$ & $k_{i}$ & $c_{i}$ & $k_{c}$ & $e_{N}$ & $\omega$ & $f_{\mathrm{ex}, 5}$ \\
\hline value & 10 & 1 & 1 & 0.3 & 8000 & 1 & $\frac{1}{5} \pi$ & $5 \cos (\omega t)$ \\
\hline
\end{tabular}

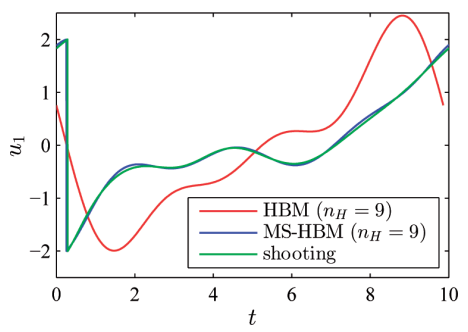

Fig. 6. Velocity of the periodic solution of the 5 DOF oscillator with impact 
periodic solution is depicted in Fig. 7 as a phase diagram which shows the existence of an accumulation point, i.e. infinitely many impacts occur during a finite time period. The HBM cannot model this effect but the MS-HBM already provides a good approximation with just a few considered harmonics and it exactly fulfils the non-penetration condition of the contact. The relation between the number of considered harmonics and the relative error of the amplitude as well as the relative calculation time are depicted in Fig 8. As in the previous examples the full shooting method is used as reference. The MSHBM provides an accuracy of less than $2 \%$ deviation of the amplitude with only one-fifth of the calculation effort. It should be noted that the reduction of the calculation effort becomes even more significant if the size of the linear subsystem increases.
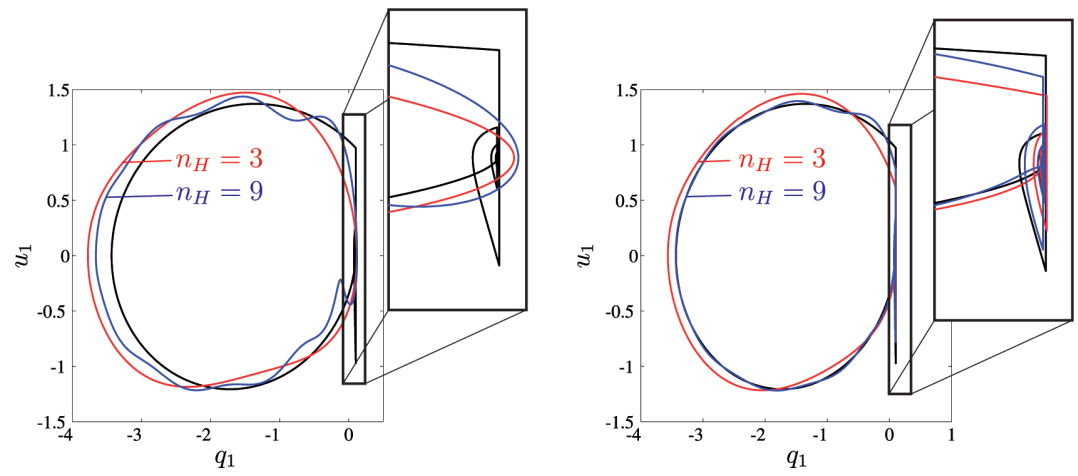

Fig. 7. Phase diagram of the nonlinear subsystem of the 50-mass oscillator calculated with 3 and 9 considered harmonics using the HBM (left), MS-HBM (right), as reference the full shooting approach is depicted in both diagrams as black curve
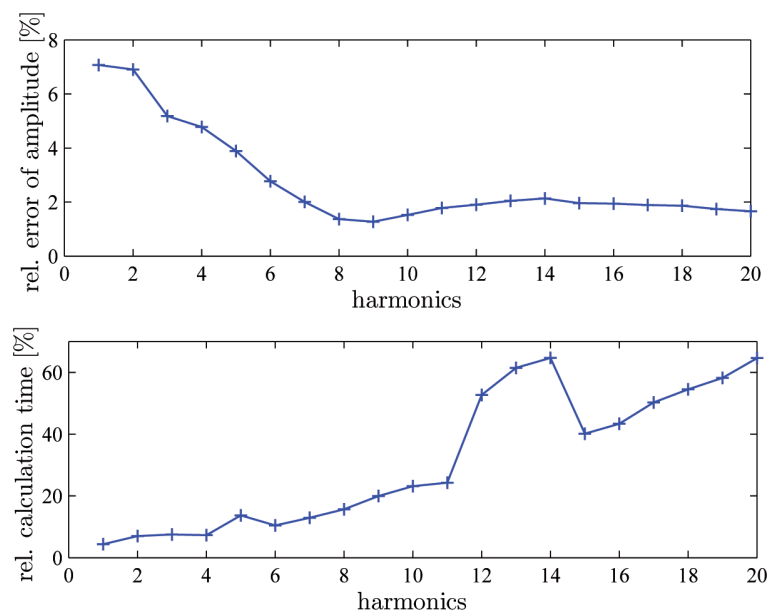

Fig. 8. Work-Precision diagram of 50-mass oscillator with unilateral constraint with the shooting method as reference 


\section{Concluding Remarks}

The presented mixed shooting-HBM approach shows good characteristics in accuracy as well as in calculation effort, at least for the investigated benchmark system with dry friction or unilateral constraint. Depending on the system size and the nonlinear characteristics the method can be a good alternative to the commonly used methods like HBM and shooting. It should be noted, that the numerical efficiency of the methods are hard to compare and that there exist alternative Harmonic Balance Methods to compute periodic solutions of systems with dry friction and impact. Further research will focus on providing a better comparison of the mixed shooting-HBM method with existing methods [10].

Manuscript received by Editorial Board, November 15, 2015; final version, June 15, 2016.

\section{REFERENCES}

[1] Magnusand K., Popp K.: Schwingungen (Vibrations), volume 3. Springer, 1961 (in German).

[2] Nayfehand A., Mook D.: Nonlinear Oscillations. John Wiley \& Sons, 2008.

[3] von Grolland G., Ewins D.: The harmonic balance method with arc-length continuation in rotor/stator contact problems. Journal of Sound and Vibration, 241:223-233, 2001.

[4] Cardona A., Coune T., Lerusse A., Geradin M.: A multiharmonic method for non-linear vibration analysis. International Journal for Numerical Methods in Engineering, 37(9):1593-1608, 1994.

[5] Ascher U.M., Mattheij R.M., Russell R.: Numerical solution of boundary value problems for ordinary differential equations, volume 13. Siam, 1994.

[6] Cameron T., Griyn J.: An alternating frequency/time domain method for cal-culating the steady-state response of nonlinear dynamic systems. Journal of applied mechanics, 56(1):149-154, 1989.

[7] Acary V., Brogliato B.: Numerical Methods for Nonsmooth Dynamical Systems: Applications in Mechanics and Electronics, volume 35. Springer Science \& Business Media, 2008.

[8] Leine R.I., Nijmeijer H.: Dynamics and Bifurcations of Nonsmooth Mechanical Systems, volume 18. Springer, Berlin, 2004.

[9] Brogliato B.: Nonsmooth Mechanics: Models, Dynamics and Control. Springer, London, 1999.

[10] Nacivet S., Pierre C., Thouverez F., Jezequel L.: A dynamic lagrangian frequency-time method for the vibration of dry-friction-damped systems. Journal of Sound and Vibration, 265(1):201-219, 2003. 


\section{Mieszana metoda strzałów i równowagi harmonicznych w zastosowaniu do systemów mechanicznych o jednostronnych więzach}

\section{Streszczenie}

W artykule przedstawiono metodę będącą połączeniem metody strzałów i metody równowagi harmonicznych zastosowaną do dużych systemów mechanicznych, w których występują lokalne nieliniowości. Standardowa metoda równowagi harmonicznych (HBM), w której aproksymuje się rozwiązanie okresowe w dziedzinie częstotliwości, jest bardzo popularna, gdyż dobrze nadaje się do dużych systemów o wielu stopniach swobody. Niemniej, jej wadą jest to, że lokalne nieliniowości nie mogą być bezpośrednio ocenione w dziedzinie częstotliwości. W standardowej metodzie HBM wykonuje się odwrotną transformację Fouriera, potem oblicza nieliniową siłę w dziedzinie czasu, a następnie wyznacza współczynniki Fouriera siły nieliniowej. Silne nieliniowości są źle reprezentowane przez obcięty szereg Fouriera, co jest wadą tej metody. W przeciwieństwie do niej, metoda strzałów działa w dziedzinie czasu i opiera się na symulacji numerycznej przebiegów czasowych. Metoda działa skutecznie gdy prawa sił są oparte na wartościach zadanych, tak jak dla tarcia suchego i innych silnie nieliniowych, pod warunkiem, że dysponuje się odpowiednim integratorem numerycznym. Metoda strzałów nie daje się jednak stosować gdy system ma wiele stanów. Proponowana metoda mieszana, strzałów i równowagi harmonicznych, łączy zalety obydwu podejść. 